\title{
Oral anticoagulation safe during cardiac device implantation
}

Investigators from the University of Barcelona, Spain, have reported that continuation of oral anticoagulation therapy is as safe as bridging to intravenous heparin in the perioperative management of high-risk patients undergoing cardiac device implantation or replacement. "This is the first randomized trial that demonstrates ... the safety of implanting devices without stopping oral anticoagulation," points out Dr Jose Maria Tolosana.

In this trial, all of the enrolled patients $(n=101)$ were at high-risk for thromboembolism $-50 \%$ had a prosthetic mitral valve and $40 \%$ had experienced a previous thromboembolic event. Patients were randomly allocated to continue daily treatment with oral acenocumarol or to switch from oral anticoagulation to intravenous heparin 4 days before surgery.
During follow-up (maximum 45 days after the procedure), there was no difference in the incidence of thromboembolic events or pocket hematomas between the two treatment groups. One of the benefits of continued oral anticoagulation was that this strategy significantly reduced the length of hospital stay. "Our study supports the implantation or replacement of devices without stopping oral anticoagulation," concludes Dr Tolosana, “....in this group of high-risk patients, one may consider implanting pacemakers as an outpatient procedure."

Alexandra King

Original article Tolosana, J. M. et al. Preparation for pacemaker implantable cardiac defibrillator implants in patients with high risk of thrombo-embolic events: oral anticoagulation or bridging with intravenous heparin? A prospective randomized trial. Eur. Heart J. doi:10.1093/ eurheartj/ehp194 